\title{
Stenotrophomonas maltophilia: resistência emergente ao SMX-TMP em isolados brasileiros. Uma realidade?
}

\section{Stenotrophomonas maltophilia: emerging resistance to TMP-SMX in Brazilian isolates. A reality?}

Luiza Souza Rodrigues'; Thais Sabato Romano Di Gioia²; Flávia Rossi³

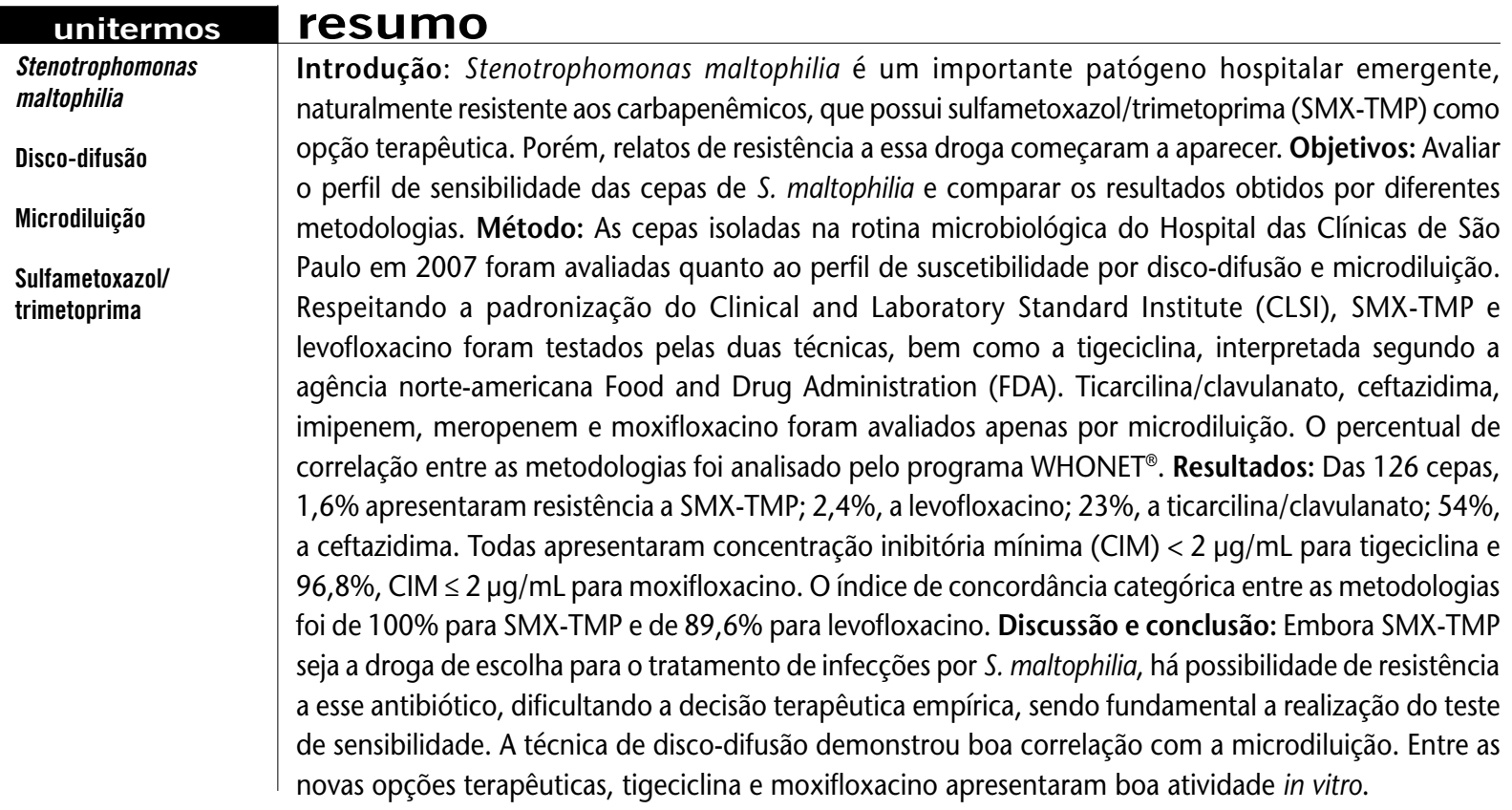

\section{abstract}

Introduction: Stenotrophomonas maltophilia is an important emerging nosocomial pathogen naturally resistant to carbapenems. Although trimethoprim/sulfamethoxazole (TMP-SMX) is commonly used as a treatment option, resistance to this drug has been recently reported. Objectives: To evaluate the susceptibility profile of S. maltophilia strains and compare the results obtained by different methods. Methods: As to susceptibility profile, all strains, which had been routinely isolated at Hospital das Clínicas, São Paulo, in 2007, were evaluated through disk diffusion and microdilution. In accordance with the Clinical and Laboratory Standard Institute (CLSI), TMP-SMX and levofloxacin were tested through both techniques, as well as tigecycline, which was interpreted in conformity with Food and Drug Administration (FDA) regulations. Ticarcillin/clavulanate, ceftazidime, imipenem, meropenem and moxifloxacin were evaluated only through microdilution. The correlation between these methods was assessed by WHONET ${ }^{\circledR}$. Results: $1.6 \%$ of 126 strains were resistant to TMP-SMX, 2.4\% to levofloxacin, 23\% to ticarcillin/clavulanate, and 54\% to ceftazidime. All strains showed minimum inhibitory concentration (MIC) $<2 \mu \mathrm{g} / \mathrm{mL}$ for tigecycline and $96.8 \% \mathrm{MIC} \leq$ $2 \mu \mathrm{g} / \mathrm{mL}$ for moxifloxacin. The correlation index between both methods was 100\% for TMP-SMX and $89.6 \%$ for levofloxacin, respectively. Discussion and conclusion: Although TMP-SMX is the standard treatment for S. maltophilia infections, there may be resistance to this antibiotic, which hinders the therapeutic approach, hence the significance of susceptibility tests. The disk diffusion technique showed a good correlation with microdilution. Among the new therapeutic options, both tigecycline and moxifloxacin presented significant activity in vitro. key words

Stenotrophomonas maltophilia

Disk diffusion

Microdilution

Trimethoprim/ sulfametoxazole

1. Pós-graduada em Patologia Clínica; biologista da Seção de Microbiologia da Divisão de Laboratório Central do Hospital das Clínicas da Faculdade de Medicina da Universidade de São Paulo (DLC-HC/FMUSP).

2. Médica patologista clínica do Serviço de Microbiologia da DLC-HC/FMUSP.

3. Doutora em Patologia Clínica; diretora médica do Serviço de Microbiologia da DLC-HC/FMUSP. 


\section{Introdução}

Stenotrophomonas maltophilia, previamente denominada Pseudomonas maltophilia e posteriormente Xanthomonas maltophilia, é atualmente a única espécie pertencente ao gênero Stenotrophomona ${ }^{(4,10,25)}$.

Caracteriza-se por ser um bacilo Gram-negativo não fermentador de glicose (BGNNFG), aeróbio, com distribuição ubíqua e baixa virulência. É considerado um agente incomum de infecções em indivíduos imunocompetentes, porém nas últimas décadas vem se destacando como um patógeno nosocomial emergente, principalmente em pacientes imunocomprometidos, com alta morbidade e mortalidade, sendo, em muitos centros, o terceiro BCNNFC de maior importância em infecções hospitalares, perdendo apenas para Pseudomonas aeruginosa e Acinetobacter

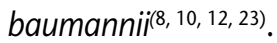

O espectro das infecções por esse agente é variado, incluindo bacteremias, pneumonias, infecções de pele e tecidos moles, endocardites, infecções no trato urinário, meningites, infecções intra-abdominais, síndromes oftalmológicas e sinusites( ${ }^{(4)}$.

Os fatores de risco para infecção por S. maltophilia incluem uso de terapia antimicrobiana de amplo espectro (carbapenêmicos, cefalosporinas de espectro estendido e fluoroquinolonas), imunossupressão, leucemias ou linfomas refratários, neutropenia pós-quimioterapia ( $<500$ células $/ \mu \mathrm{L})$, mucosite do trato gastrointestinal (TGI) secundária à quimioterapia, radioterapia, doença enxerto versus hospedeiro, permanência prolongada em unidade de terapia intensiva (UTI), ventilação mecânica prolongada (mais de sete dias) e uso de dispositivos intravasculares ${ }^{(4-7,20,22)}$. A presença de cateter venoso central e ventilador mecânico funciona como porta de entrada e superfície de adesão desse microrganismo, que tem como uma de suas principais vantagens adaptativas a habilidade de formar biofilme em materiais constituídos por poliestireno. A produção da matriz extracelular do biofilme é crucial para aderência da bactéria, proteção contra o sistema imunológico do hospedeiro e atividade antimicrobiana ${ }^{(7)}$.

$\mathrm{O}$ isolamento laboratorial de cepas de S. maltophilia em amostras biológicas nem sempre traduz infecção, pois o TGl, as vias aéreas e a pele podem ser sítios de colonização no indivíduo. A colonização desses sítios pode estar relacionada com estadia prolongada em UTI, uso de traqueostomia, exposição prévia com fluorquinolona e terapia prolongada com carbapenem ou cefalosporina de quarta geração(4, 21).
A relação do uso dos betalactâmicos com o aumento da incidência de infecções por S. maltophilia ainda é assunto controverso na literatura. Em um estudo epidemiológico realizado no Hospital das Clínicas da Faculdade de Medicina da Universidade de São Paulo (HC/FMUSP) no período de 1999 a 2006, com o objetivo de avaliar o efeito do uso de carbapenêmicos e cefalosporinas de quarta ou terceira geração (com ação antipseudomonas) e a associação dessas drogas ao aumento da incidência desse agente em infecções de corrente sanguínea, não foi observado aumento significativo na taxa de incidência ${ }^{(18)}$.

O tratamento de escolha para as infecções causadas por esse microrganismo é a associação sulfametoxazol/ trimetoprima (SMX-TMP) em dose similar à utilizada no tratamento de pneumonia por Pneumocystis jiroveci ( $\geq 15 \mathrm{mg} / \mathrm{kg} / \mathrm{dia}$ ), pois diversos mecanismos de resistência estão presentes minimizando a atividade de outras drogas, como carbapenêmicos, cefalosporinas e aminoglicosídeos $(1,2,8,13,16,17,22)$.

A emergência de cepas de $S$. maltophilia resistentes a SMX-TMP associada à escassez de trabalhos clínicos utilizando drogas alternativas tornam a escolha terapêutica um desafio. Esse fato vem ganhando destaque na literatura, com taxas mundiais de resistência que variaram de $2 \%$ a $10 \%$, sendo os maiores percentuais encontrados em países da Europa ${ }^{(8,22)}$.

A técnica padrão para a avaliação do perfil de sensibilidade aos antimicrobianos (PSA) desse microrganismo é a microdiluição em ágar ou caldo, para a qual o Clinical and Laboratory Standard Institute (CLSI) padronizou valores de interpretação dos resultados para seis drogas: SMX-TMP, levofloxacino, cloranfenicol, ticarcilina/clavulanato, ceftazidima e minociclina. Esse documento também dispõe de interpretação pela técnica de disco-difusão, porém somente para três antibióticos (SMX-TMP, levofloxacino e minociclina), devido à baixa correlação da metodologia padrão com as demais drogas.

Os objetivos deste trabalho foram avaliar o perfil de sensibilidade das cepas de S. maltophilia isoladas no complexo hospitalar do HC/FMUSP e comparar os resultados obtidos pelas metodologias laboratoriais disponíveis.

\section{Métodos}

Durante o ano de 2007, todas as cepas de $S$. maltophilia, isoladas de amostras biológicas na rotina do Laboratório de Microbiologia da Divisão de 
Laboratório Central (DLC) do HC/FMUSP, foram armazenadas em microtubos com uma gota de glicerina para $1 \mathrm{~mL}$ de caldo triptona de soja (TSB) e guardadas em freezer a $-70^{\circ} \mathrm{C}$. No ano seguinte, essas cepas foram reconstituídas, confirmadas quanto à identificação com testes bioquímicos, e uma nova avaliação do perfil de sensibilidade in vitro foi realizada por meio das metodologias de disco-difusão (Kirby-Bauer) e microdiluição em caldo, conforme orientações do fabricante (MicroScan ${ }^{\circledast}$, Siemens).

Os antibióticos testados pela técnica de disco-difusão foram SMX-TMP, levofloxacino e tigeciclina $\left(\right.$ Oxoid $\left.^{\circledR}\right)$. As cepas não sensíveis a tigeciclina foram testadas também pela metodologia de E-test ${ }^{\circledR}$ (AB-Biodisk) e microdiluição.

O painel de antibióticos avaliado por microdiluição (MicroScan ${ }^{\circledast}$, Siemens) e suas respectivas diluições foram: SMX-TMP $(2 / 38 \mu \mathrm{g} / \mathrm{mL})$, levofloxacino (2 e $4 \mu \mathrm{g} / \mathrm{mL})$, ticarcilina/clavulanato $(12 / 2$ e $64 / 2 \mu \mathrm{g} / \mathrm{mL})$, ceftazidima (8 e $16 \mu \mathrm{g} / \mathrm{mL})$, imipenem $(4$ e $8 \mu \mathrm{g} / \mathrm{mL})$, meropenem (4 e $8 \mu \mathrm{g} / \mathrm{mL})$, moxifloxacino (2 e $4 \mu \mathrm{g} / \mathrm{mL}$ ) e tigeciclina $(0,025$ a $16 \mu \mathrm{g} / \mathrm{mL})$, com leitura manual após 18 a 24 horas de incubação a $35^{\circ} \mathrm{C} \pm 2^{\circ} \mathrm{C}$.

Os critérios para a interpretação dos resultados basearam-se no documento CLSI M100-S18, com exceção de tigeciclina e moxifloxacino. Para tigeciclina, foram utilizados os pontos de corte do Food and Drug Administration (FDA) definidos para enterobac- térias, sendo a concentração inibitória mínima (CIM) $\leq 2 \mu \mathrm{g} / \mathrm{mL}$, $=4 \mu \mathrm{g} / \mathrm{mL} \mathrm{e} \geq 8 \mu \mathrm{g} / \mathrm{mL}$, consideradas sensível, intermediária e resistente, respectivamente; e para moxifloxacino foram utilizados os pontos de corte do CLSI do levofloxacino.

O percentual de concordância categórica dos resultados obtidos entre as duas metodologias foi analisado no programa estatístico WHONET ${ }^{\circledR}$. A correlação entre os métodos foi avaliada de acordo com o tipo de erro categórico, conforme estabelecido pelo CLSI. Erro gravíssimo é definido como falsa sensibilidade, erro grave é falsa resistência e erro leve é aquele no qual a categoria discrepante passa de sensível para intermediária, de resistente para intermediária ou vice-versa. Os erros leves $(\leq 10 \%)$, graves $(\leq 3 \%)$ e gravíssimos $(\leq 1,5 \%)$ são os níveis definidos pelo CLSI como aceitáveis para correlação de métodos ${ }^{(14)}$.

\section{Resultados}

Das 126 cepas de S. maltophilia incluídas no estudo, 60 $(47,6 \%)$ foram isoladas de sangue e ponta de cateter; 46 $(36,5 \%)$, de amostras provenientes do trato respiratório (lavado bronquicoalveolar, secreção traqueal, escarro e secreção de orofaringe); 15 (11,9\%), de outros materiais, como feridas cirúrgicas, abscessos e secreções; e 5 (4\%), de líquidos nobres (líquido ascítico, líquido pleural e liquor), conforme Figura 1.

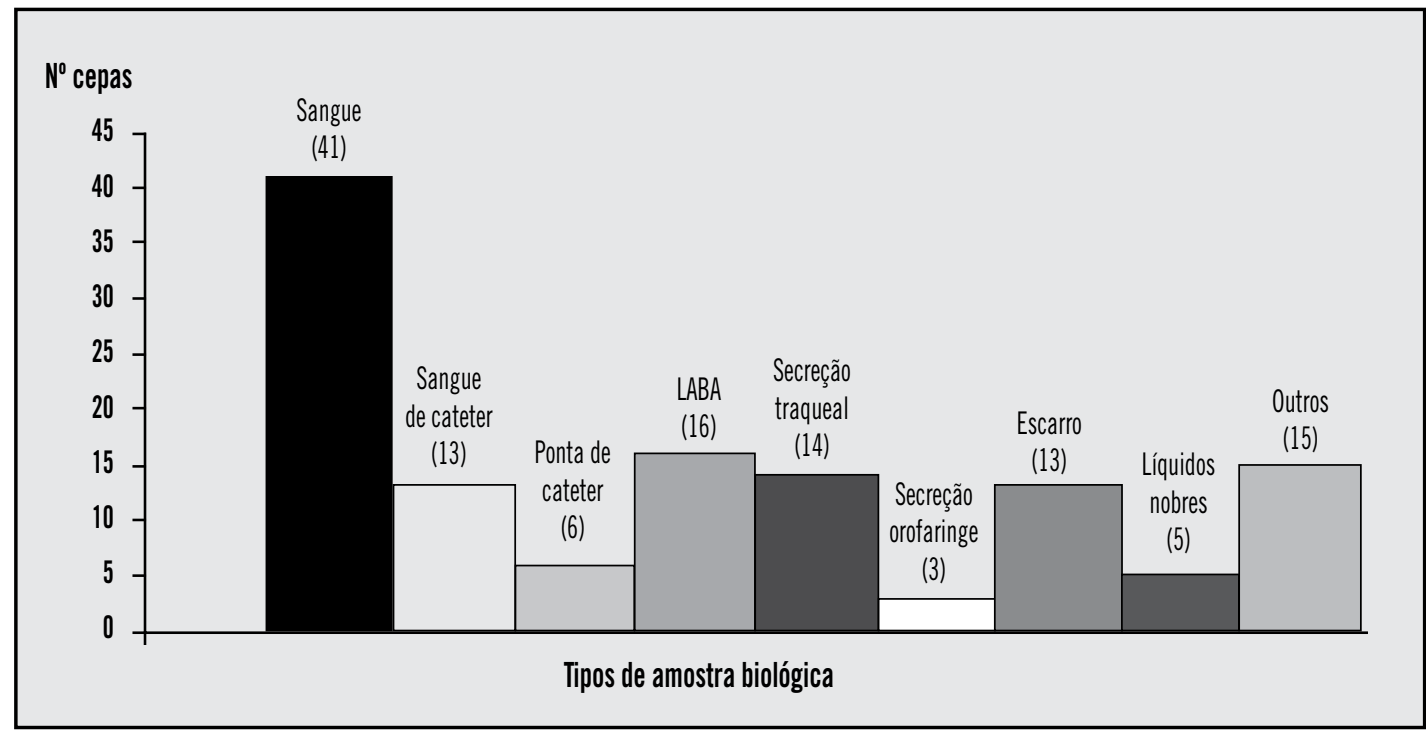

Figura 1 - Distribuição das cepas de S. maltophilia isoladas, segundo o tipo de material biológico (DLC-HC/FMUSP, 2007) DLC- HC/FMUSP: Divisão de Laboratório Central do Hospital das Clínicas da Faculdade de Medicina da Universidade de São Paulo; LABA: lavado broncoalveolar. 
No estudo do perfil das drogas padronizadas pelo CLSI por microdiluição, obtivemos as taxas de resistência de $1,6 \%$ a SMX-TMP, $2,4 \%$ a levofloxacino, $23 \%$ a ticarcilina/ clavulanato e $54 \%$ a ceftazidima. As duas cepas resistentes a SMX-TMP também foram resistentes a levofloxacino, ticarcilina/clavulanato e ceftazidima, com $100 \%$ de sensibilidade apenas a tigeciclina. Moxifloxacino apresentou $96,8 \%$ de sensibilidade com CIM $\leq 2 \mu \mathrm{g} / \mathrm{mL}$. Todas as cepas apresentaram $\mathrm{CIM} \geq 8 \mu \mathrm{g} / \mathrm{mL}$ a imipenem e meropenem (Tabela 1).

Ao comparar os resultados categóricos entre as metodologias utilizadas, o índice de concordância para SMX-TMP foi de $100 \%$ e para levofloxacino, $89,7 \%$ (Figuras 2 e 3 ).

Entre as discrepâncias categóricas encontradas para levofloxacino, $8 \%$ foram de erros leves e 2,4\%, erros graves. Em relação a tigeciclina, quando testada por disco-difusão, oito cepas apresentaram-se não sensíveis, porém, quando foram avaliadas por microdiluição, apresentaram CIM abaixo de $2 \mu \mathrm{g} / \mathrm{mL}$ e, portanto, são sensíveis segundo os critérios do FDA (Tabela 2).

\section{Discussão}

O perfil de sensibilidade apresentado pelos isolados de S. maltophilia neste estudo foi compatível com o característico desse agente, apresentando baixos níveis de sensibilidade aos antibióticos betalactâmicos e betalactâmicos associados a inibidores de betalactamase e melhor sensibilidade a SMX-TMP e fluoroquinolonas ${ }^{(1,2,4,17)}$. Embora SMX-TMP seja o antibiótico de escolha nas infecções por S. maltophilia, a emergência dessa resistência vem ganhando destaque na literatura, variando de $2 \%$ no Canadá e na América Latina a $10 \%$ na Europa. Neste estudo, encontramos 1,6\% (duas cepas) de resistência a esse antimicrobiano, índice similar ao encontrado em dados brasileiros publicados em 2004, que detectaram 1,5\% de cepas não sensíveis a esse antibiótico(8,15).

A possibilidade da resistência a SMX-TMP, apesar de não ser expressiva e não ter demonstrado aumento significativo nos últimos anos, pode dificultar a decisão terapêutica empírica nas infecções graves, visto que a

\section{Perfil de suscetibilidade das cepas de S. maltophilia $(n=126)$ por microdiluição e disco-difusão}

Tabela 1 (DLC-HC/FMUSP)

\begin{tabular}{l|c|c|c|ccc}
\hline & \multicolumn{3}{|c|}{ Microdiluição } & \multicolumn{3}{c}{ Disco-difusão } \\
\cline { 2 - 7 } & $\mathbf{S} \%$ & $\mathbf{1} \%$ & $\mathbf{R} \%$ & $\mathbf{S} \%$ & $\mathbf{I} \%$ & $\mathbf{R} \%$ \\
\hline Sulfametoxazo//trimetoprima & 98,4 & 0 & 1,6 & 98,4 & 0 & 1,6 \\
\hline Levofloxacino & 88,9 & 8,7 & 2,4 & 88,1 & 5,5 & 6,4 \\
\hline Ticarcilina/clavulanato & 33,3 & 44,7 & 29 & NSA & NSA & NSA \\
\hline Ceftazidima & 34,1 & 11,9 & 68 & NSA & NSA & NSA \\
\hline *Moxifloxacino & 96,8 & 0,8 & 2,4 & NSA & NSA & NSA \\
\hline
\end{tabular}

*Utilizado ponto de corte (CLSI) do levofloxacino.

DLC-HC/FMUSP: Divisão de Laboratório Central do Hospital das Clínicas da Faculdade de Medicina da Universidade de São Paulo; S: sensivel; I: intermediária; R: resistente; NSA: não se aplica; CLSI: Clinical and Laboratory Standard Institute.

\section{Perfil de suscetibilidade das cepas de $S$. maltophilia $(n=126)$ a tigeciclina pelas técnicas de}

Tabela 2 disco-difusão, E-test ${ }^{\circledR}$ e microdiluição (DLC-HC/FMUSP)

\begin{tabular}{l|c|c|c|c|}
\hline \multicolumn{1}{|c|}{ Metodologia } & $\begin{array}{c}N^{0} \text { de cepas } \\
\text { avaliadas }\end{array}$ & $\mathbf{S}$ & Perfil de suscetibilidade & $\mathrm{R}$ \\
\hline Disco-difusão & 126 & 118 & 5 & 3 \\
\hline${ }^{*}$ E-test $^{\circledR}$ & 8 & 1 & 2 & 5 \\
\hline${ }^{*}$ Microdiluição & 8 & 8 & 0 & 0 \\
\hline
\end{tabular}

${ }^{*}$ E-test ${ }^{\boxplus}$ e microdiluição realizados para a confirmação das oito cepas não sensíveis a tigeciclina pela metodologia de disco-difusão.

DLC-HC/FMUSP: Divisão de Laboratório Central do Hospital das Clínicas da Faculdade de Medicina da Universidade de São Paulo; S: sensivel; I: intermediária; $R$ : resistente. 


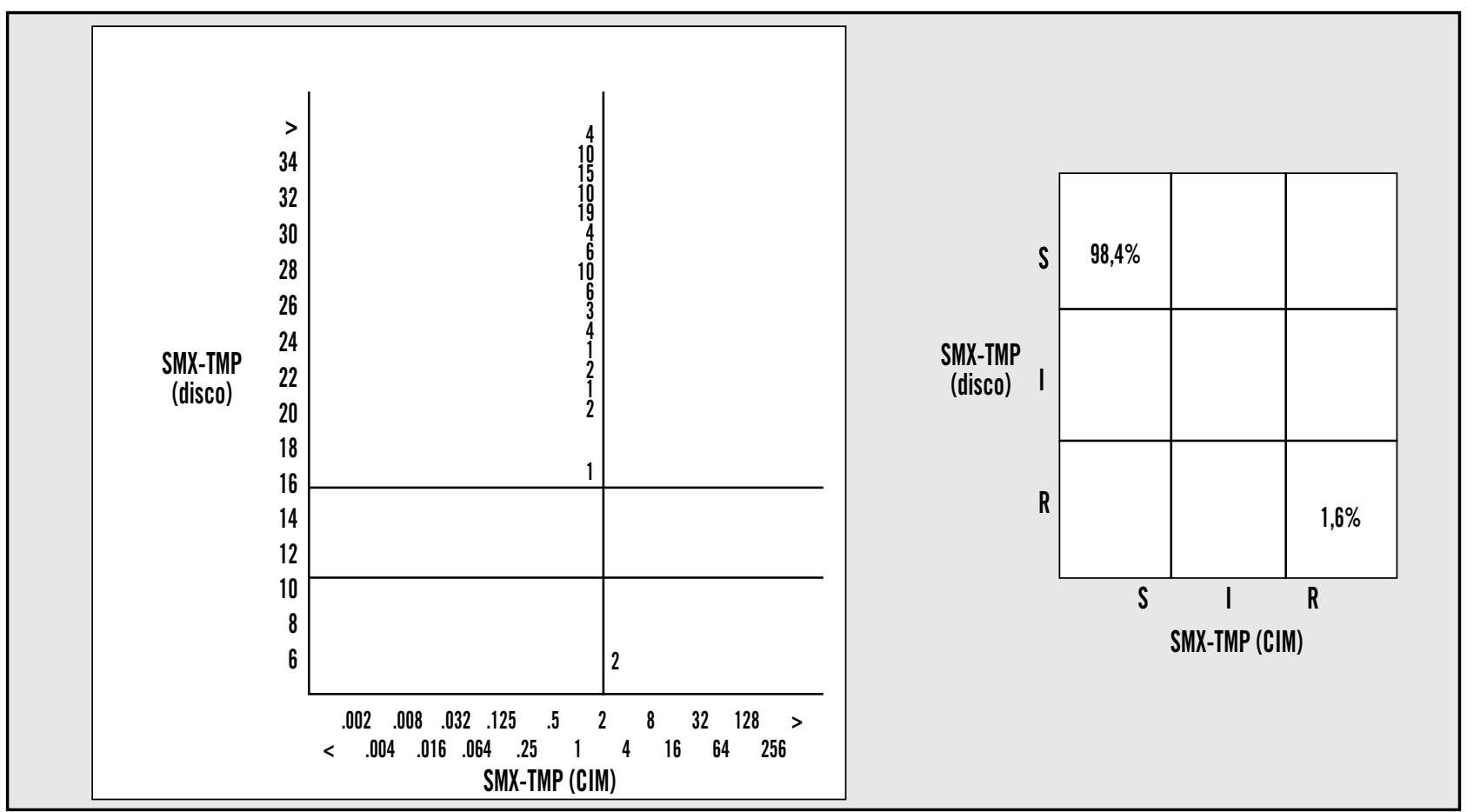

Figura 2 - Comparação entre as categorias obtidas por microdiluição (CIM) e disco-difusão (disco) para SMX-TMP ( $\mathrm{n}=126$, S. maltophilia) CIM: concentração inibitória mínima; SMX-TMP: sulfametoxazol/trimetoprima; S: sensível; I: intermediária: R: resistente.
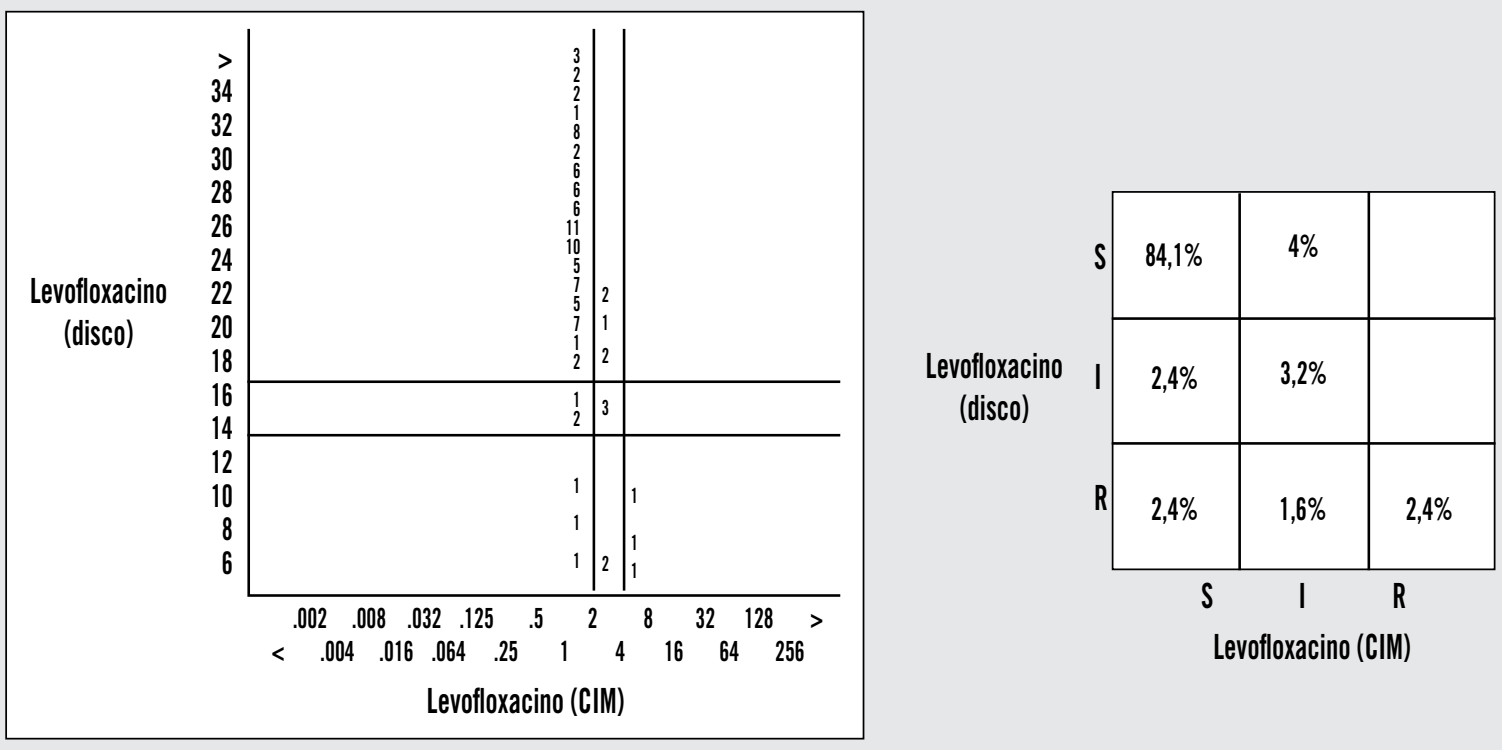

Figura 3 - Comparação entre as categorias obtidas por microdiluição (CIM) e disco-difusão (disco) para levofloxacino ( $\mathrm{n}=126$, S. maltophilia) CIM: concentração inibitória mínima; S: sensível; I: intermediária: R: resistente.

resistência a essa droga pode estar associada à resistência a outros antibióticos considerados ativos contra esse patógeno ${ }^{(19)}$. O antibiograma dos isolados deve ser realizado rotineiramente para monitorar a emergência dessas resistências. Os mecanismos envolvidos na resistência a SMX-TMP ainda não estão bem esclarecidos, mas acredita-se que a aquisição de integrons de classe 1 , contendo o gene sul1 na sua extremidade 3 não traduzida, contribua para esse perfil, bem como a capacidade de formar biofilme que, apesar de não ser, de fato, um mecanismo de resistência, é capaz de dificultar a atividade da droga sobre a bactéria ${ }^{(24)}$. Além da resistência a SMX-TMP, também há a possibilidade de intolerância a essa droga, tornando-se fundamental a análise in vitro de outras que alternativamente possam ser utilizadas no tratamento das infecções por esse agente. 
Em relação à esperada baixa sensibilidade aos betalactâmicos, isso se deve ao fato de a membrana externa da S. maltophilia ser impermeável a essa classe de antibióticos, e menos de $5 \%$ deles possuem capacidade de penetrar na membrana. Há descrição de atividades variadas de cefalosporinas e combinações de betalactâmicos com inibidores de betalactamases, como ceftazidima, cefepima, ticarcilina/ clavulanato e piperacilina/tazobactam.

Em nossa instituição, detectamos $54 \%$ de resistência a ceftazidima e $23 \%$, a ticarcilina/clavulanato, enquanto na literatura esses antibióticos possuem diferentes dados de sensibilidade, variando de $25 \%$ a $92 \%$ e de $10 \%$ a $64 \%$, respectivamente ${ }^{(8,9)}$. A baixa atividade dos carbapenêmicos, característica desse agente, é esperada associando-a à alta eficiência na atividade de duas betalactamases (L1 e L2) induzíveis, isto é, que têm sua expressão estimulada quando a célula é exposta aos agentes betalactâmicos e que podem agir isoladamente ou em conjunto, destruindo a atividade desse grupo de antibióticos. L1 é uma metalobetalactamase (enzima que requer $\mathrm{Zn}^{2+}$ como cofator), cuja característica é a habilidade de hidrolisar todos os betalactâmicos, com exceção dos monobactans (aztreonam), não sendo suscetível aos inibidores de betalactamase, e exibindo, predominantemente, atividade de carbapenemase. L2 é uma serinobetalactamase (enzima que contém serina no seu sítio ativo), que hidrolisa os monobactans, inibe-se por meio dos inibidores de betalactamase e exibe atividade de cefalosporinase $^{(2,}{ }^{21)}$. Assim, mesmo que seja encontrada eventualmente baixa CIM in vitro para imipenem ou meropenem, eles não deverão ser reportados como sensíveis.

As novas fluoroquinolonas são consideradas boa opção terapêutica, com destaque atualmente para levofloxacino e moxifloxacino, que apresentaram boa atividade in vitro nas cepas isoladas em nossa instituição. Estudos anteriores demonstraram baixa atividade do ciprofloxacino contra essa espécie bacteriana, além de grande discrepância de concordância categórica entre as metodologias disco-difusão e microdiluiçãa ${ }^{(3,22)}$.

Recentemente, surgiram novas opções terapêuticas, em uso combinado ou não, porém sua interpretação e seu uso clínico devem ser criteriosos ${ }^{(1,11,21,22)}$. É o caso, por exemplo, da tigeciclina, que, apesar de ainda não possuir interpretação laboratorial e grande número de ensaios clínicos, apresentou elevada eficiência in vitro diante das cepas de S. maltophilia em nosso estudo, informação concordante com dados recentes reportados na literatura, com taxas de suscetibilidade que variaram de $87 \%$ a $100 \%(21,22)$. Neste estudo, apenas as cepas resistentes ou intermediárias a tigeciclina (oito cepas) por disco-difusão foram também avaliadas por microdiluição e E-test ${ }^{\circledR}$, pois é sabido que esse antibiótico, quando testado por metodologias de difusão em ágar, pode sofrer interferência do manganês contido no ágar Mueller-Hinton, podendo gerar resultados falsamente intermediários ou resistentes, o que também foi verificado(11).

Artigos recentes relatam a imprecisão do antibiograma por disco-difusão em relação à microdiluição, entretanto, essa observação está relacionada somente com antibióticos não padronizados atualmente pelo $\mathrm{CLSI}^{(3,22-24)}$.

Em nosso estudo, houve boa correlação das metodologias para as drogas padronizadas pelo CLSI, podendo, então, o método de disco ser facilmente aplicado nos laboratórios de rotina, guiando, assim, uma terapêutica mais segura nas infecções por S. malthophilia. Embora a técnica de disco-difusão seja apenas qualitativa, seus resultados categóricos são confiáveis e de fácil execução, associados a menor custo quando em comparação com a microdiluição. $O$ teste da tigeciclina por disco-difusão é exceção, conforme descrevemos anteriormente, e esses resultados, quando sensíveis, podem ser considerados sem necessidade de confirmação.

Outras opções terapêuticas, como colistina e polimixina B, ressurgiram como antibióticos alternativos diante das cepas multirresistentes de S. maltophilia, mas há dificuldades técnicas em suas avaliações in vitro, e não testamos essas drogas em nosso estudo(15).

\section{Conclusão}

A emergência de resistência a SMX-TMP também é uma realidade em hospitais brasileiros e deve ser monitorada para garantir a melhor escolha terapêutica em infecções por S. maltophilia.

Determinar um perfil de sensibilidade individualizado e monitorar tendências de emergência de resistência são atributos importantes do laboratório de microbiologia, principalmente para microrganismos intrinsecamente resistentes às diversas classes de drogas, como é o caso da S. maltophilia. Resistências atípicas ou emergentes devem ser confirmadas e comunicadas ao corpo clínico e às comissões de controle de infecção hospitalar.

\section{Agradecimentos}

À equipe do Laboratório de Microbiologia da DLC-HC/ FMUSP pelo trabalho realizado, pela rotina, pela disponibilização dos dados e pela estrutura para a realização deste estudo. 


\section{Referências}

1. ALONSO, A.; MARTINEZ, J. L. Expression of multidrug efflux pumps SmeDEF by clinical isolates of Stenotrophomonas maltophilia. Antimicrob Agents Chemother, v. 45, p. 1879-81, 2001.

2. AVISON, M. B. et al. Differential regulation of $L 1$ and $L 2$ betalactamase expression in Stenotrophomonas maltophilia. J Antimicrob Chemother, v. 49, p. 387-9, 2002.

3. CARROLL, K. C. et al. Comparison of various in vitro susceptibility methods for testing Stenotrophomonas maltophilia. Diagn Microbiol Infect Dis, v. 32, p. 229-35, 1998.

4. CORZO-DELGADO, J. E.; GOMEZ-MATEOS, J. M. Stenotrophomonas maltophilia, an increasingly important nosocomial pathogen. Enferm Infecc Microbiol Clin, v. 24, p. 1-3, 2006.

5. DEL TORO, M. D. et al. Clinical epidemiology of Stenotrophomonas maltophilia colonization and infection: a multicenter study. Medicine (Baltimore), v. 81, p. 228-39, 2002.

6. DEL TORO, M. D. et al. Epidemiology, clinical features and prognosis of infections due to Stenotrophomonas maltophilia. Enferm Infecc Microbiol Clin, v. 24, p. 4-9, 2006.

7. DI BONAVENTURA, G. et al. Effect of environmental factors on biofilm formation by clinical Stenotrophomonas maltophilia isolates. Folia Microbiol (Praha), v. 52, p. 86-90, 2007.

8. GALES, A. C. et al. Emerging importance of multidrugresistant Acinetobacter species and Stenotrophomonas maltophilia as pathogens in seriously ill patients: geographic patterns, epidemiological features, and trends in the SENTRY Antimicrobial Surveillance Program (1997-1999). Clin Infect Dis, v. 32, suppl. 2, p. S104-13, 2001.

9. GÜLMEZ, D. et al. Comparison of different antimicrobial susceptibility testing methods for Stenotrophomonas maltophilia and results of synergy testing. J Infect Chemother, v. 16, n. 5, p. 322-8, 2010.

10. JULIET, L. C.; FERNANDEZ, V. A. Stenotrophomonas maltophilia. Rev Chilena Infectol, v. 23, p. 247-8, 2006.

11. MAZARRASA, C. F. et al. High concentrations of manganese in Mueller-Hinton Agar increase MICs of tigecycline determined by Etest. Journal of Clinical Microbiology, v. 47, p. 827-9, 2009

12. MCGOWAN, J. E. Resistance in nonfermenting gramnegative bacteria: multidrug resistance to the maximum. Am J Infect Control, v. 3, p. S29-37, discussion p. S64-73, 2006.

13. MULDER, R. H.; FARNHAM, S. M.; GRINIUS, B. Evaluating antimicrobial susceptibility test systems, In: ISENBERG, H. D. (Ed.). Clinical microbiology procedures handbook. Washington, DC: American Society for Microbiology, 1992, p. 5.23.1-5.23.15.

14. NCCLS. Development of in vitro susceptibility testing and quality control parameters. Approved standard, $2^{\text {nd }}$ ed. NCCLS document M23-A2. Wayne, PA, 2001.

15. NICODEMO, A. C. et al. In vitro susceptibility of Stenotrophomonas maltophilia isolates: comparison of disc diffusion, Etest and agar dilution methods. J Antimicrob Chemother, v. 53, p. 604-8, 2004.

16. OKAZAKI, A.; AVISON, M. B. Aph(3)-Ilc, an aminoglycoside resistance determinant from Stenotrophomonas maltophilia. Antimicrob Agents Chemother, v. 51, p. 359-60, 2007.

17. PATHMANATHAN, A.; WATERER, G. W. Significance of positive Stenotrophomonas maltophilia culture in acute respiratory tract infection. Eur Respir J, v. 25, p. 911-4, 2005.

18. PAEZ, J. G. et al. Trends in Stenotrophomonas maltophilia bloodstream infection in relation to usage density of cephalosporins and carbapenems during 7 years. Infection Control and Hospital Epidemiology, v. 29, p. 989-90, 2008.

19. POMPILIO, A. etal. Factors associated with adherence to and biofilm formation on polystyrene by Stenotrophomonas maltophilia: the role of cell surface hydrophobicity and motility. FEMS Microbiol Lett, v. 287, p. 41-7, 2008.

20. ROSSI, F.; ANDREAZZI, D. Overview of tigecycline and its role in the era of antibiotic resistance. The Brazilian Journal of Infectious Diseases, v. 10, p. 203-16, 2006.

21. SAFDAR, A.; ROLSTON, K. V. Stenotrophomonas maltophilia: changing spectrum of a serious bacterial pathogen in patients with cancer. Clinical Infectious Diseases, v. 45, p. 1602-9, 2007.

22. SENOL, E. Stenotrophomonas maltophilia: the significance and role as a nosocomial pathogen. J Hosp Infect, v. 57, p. 1-7, 2004.

23. TOLEMAN, M. A. et al. Global emergence of trimethoprim/ sulfamethoxazole resistance in Stenotrophomonas maltophilia mediated by acquisition of sul genes. Emerg Infect Dis, v. 13, p. 559-65, 2007.

24. TRAUB, W. H.; LEONHARD, B.; BAUER, D. Antibiotic susceptibility of Stenotrophomonas (Xanthomonas) maltophilia: comparative (NCCLS criteria) evaluation of antimicrobial drugs with the agar dilution and the agar disk diffusion (Bauer-Kirby) tests. Chemotherapy, v. 44, p. 164-73, 1998.

25. WANG, W. S. et al. Stenotrophomonas maltophilia bacteremia in adults: four years experience in a medical center in northern Taiwan. J Microbiol Immunol Infect, v. 37, p. 359-65, 2004.

\begin{tabular}{l|l} 
& Endereço para correspondência \\
\hline & Luiza Souza Rodrigues \\
& Av. Dr. Eneas de Carvalho Aguiar, 155, PAMB, $2^{\circ}$ andar/Bloco 3 \\
& Cerqueira César \\
& CEP: 05403-900 - São Paulo-SP \\
& Tel.: (11) 3069-6348 \\
& e-mail: luizabiomed@hotmail.com
\end{tabular}

